\title{
LA ADQUISICIÓN DE COMPETENCIAS PARA LA VIDA DIARIA. UN ESTUDIO DE CASOS. DIFERENCIAS DE SEXO ENTRE PERSONAS CON DISCAPACIDAD
}

\author{
MARTA LIESA ORUS \\ Universidad de Zaragoza \\ ELIAS VIVED CONTE \\ Universidad de Zaragoza
}

Recibido: 01/11/2008

Aceptado: 03/03/2009

\section{Introducción}

Este artículo describe una investigación que se está desarrollando desde la Facultad de Ciencias Humanas y de la Educación de Huesca. Universidad de Zaragoza. Se trata de que en un piso de estudiantes jóvenes con discapacidad convivan estudiantes de magisterio. Uno de los objetivos del proyecto es que las personas con discapacidad aprendan habilidades para vivir de manera independiente. Nos interesa conocer si la adquisición de estas destrezas está condicionada por la variable sexo. Es decir, si la actitud hacia las tareas del hogar es similar independientemente del sexo que tenga el usuario/a.

La metodología de investigación empleada ha sido la cualitativa, en concreto, el método de casos como herramienta de investigación en las Ciencias Sociales. La recogida de información básica consistió en observaciones sistemáticas, cuestionarios sobre calidad de vida, autodeterminación y habilidades para la vida diaria que se pasaron a las personas con discapacidad que convivieron en el piso. Dichos cuestionarios se realizaron al inicio de su estancia y al final de la misma. 


\section{Fundamentación teórica}

El proyecto «vida independiente», está dentro de los programas de transición a la vida independiente y el objetivo prioritario de estos programas dirigidos a personas con discapacidad intelectual es aumentar la independencia y mejorar su calidad de vida. Para ello, un equipo de educadores les ayudamos a realizar las tareas domésticas, a cuidar su higiene personal y a integrarse en la comunidad, en definitiva, a desenvolverse en todos los aspectos de la vida diaria.

Estos programas no son de asistencia total o una alternativa para todos, sino modelos en el proceso de evolución hacia nuevas propuestas. Su éxito radica en la elección de un entorno normalizado (una vivienda), la voluntariedad de los participantes y el apoyo continuado de profesionales, estudiantes, amigos y familiares.

En su mayoría, las personas con discapacidad residen en el domicilio familiar o en residencias e instituciones gestionadas por una entidad. Los programas de transición les permiten salir de este entorno para convivir en una vivienda, solos, con otros jóvenes o con personas de características similares. Estos programas les permiten mejorar su autonomía, mejorar sus potencialidades, aumentar su satisfacción personal y su independencia.

Los servicios que se prestan a quienes participan en un programa de transición a la vida independiente dependen del grado de discapacidad que presentan. Se les ayuda a realizar las tareas del hogar, a cuidar la higiene personal o a realizar la compra, entre otras cosas. Un equipo de educadores se encarga de visitar las viviendas, una o varias veces por semana. Y el día a día lo pasan con estudiantes universitarios con los que conviven en el piso. El objetivo es que las personas con discapacidad sean capaces de vivir en el futuro de manera independiente. Al ser un programa de transición, lo habitual es reducir el apoyo conforme avanza el proyecto. La experiencia se plantea como un entrenamiento real: «A vivir se aprende viviendo, nadie se va a talleres a aprender a vivir sólo».

Hemos podido observar que los jóvenes acuden al proyecto sin saber hacer casi ninguna de las tareas del hogar y de las tareas para la vida independiente, notamos la sobreprotección que fundamentalmente los chicos con discapacidad sufren en sus familias, muchos cuando acuden al piso nunca han puesto una lavadora, se han cosido un botón, han hecho una cama, han cocido una verdura, etc.

También hemos podido observar que la implicación de las familias y el creer que este proyecto es importante para sus hijas/os es fundamental; si una familia no se implica, lo que se ha aprendido en el proyecto a los pocos días 
de que el joven ha vuelto al hogar familiar ya se ha perdido y no se ha podido generalizar. Observamos en algunos casos que después de las vacaciones de Navidad o Semana Santa los resultados obtenidos bajan bastante.

Hay que mentalizar a las familias de que algunos chicas/os con discapacidad intelectual pueden llegar a vivir de manera autónoma si les damos los servicios y apoyos que precisan en cada momento y si las familias colaboran en la generalización de los aprendizajes de sus hijas e hijos, y tienen positivas expectativas en ellos. Sabemos que este proyecto es muy novedoso y que necesitamos previamente formar a las familias para que los resultados que queremos conseguir con esas estancias de los jóvenes en el piso se generalicen luego a la vida diaria y puedan llegar a vivir algún día solos o con unos apoyos mínimos.

Haciendo un repaso histórico vemos cómo la concepción y las prácticas sociales en relación a las personas con discapacidad intelectual han variado a lo largo de la historia. El concepto de retraso mental y su tratamiento ha sufrido un cambio sustancial en este último siglo. Anteriormente nadie se planteaba los programas de transición a la vida adulta para las personas con discapacidad. Actualmente, nos encontramos ante un cambio muy importante en la forma de entender el retraso mental, que ha pasado de visiones deficitarias e individuales a modelos ecológicos, que entienden las necesidades de las personas desde la oferta de servicios y apoyos que se proporcionan, en nuestro caso un piso de entrenamiento dónde los jóvenes aprenden a vivir de manera independiente.

El concepto de discapacidad intelectual es un concepto social. Surge de la relación entre el modo en que una sociedad se organiza y articula y su forma de comprender las diferencias, las actitudes que manifiesta y las respuestas sociales que genera ante ello. En consecuencia, este concepto se caracteriza por ser dinámico y cambiante en la medida en que la sociedad va evolucionando ${ }^{1}$.

A finales de los años 70 y en la década de los 80 se producen momentos de cambio, en un contexto de confusión por la pluralidad de enfoques existentes y métodos de intervención. Dos nuevos modelos surgen en este contexto: el primero, un modelo educativo que se presentó en 1978 en el Informe Warnock sobre integración escolar y que incorporó el concepto de necesidades educativas especiales. El segundo, el modelo propuesto por la OMS en 1982, que establece un sistema de comprensión a través de la Clasificación Internacional de Deficiencias, Discapacidades y Minusvalías; en él se rompe con

1. SCherenberger, R. C. Historia del retraso mental. San Sebastián, SIIS, 1984. Agudo, A. L. Historia de las deficiencias. Madrid, Escuela Libre Editorial,1995. 
las categorías hasta entonces establecidas y se centra la atención en el ajuste entre las personas y sus entornos, y por tanto en los servicios y necesidades de apoyo de las personas.

En 1992 la Asociación Americana por el Retraso Mental (AAMR) propone una nueva definición sobre retraso mental que trata de poner fin a la discusión sobre la validez del criterio de inteligencia, de conectar la definición con algunos modelos más en desarrollo de la psicología y con otras perspectivas como la sociológica. La nueva propuesta no sólo trata de avanzar en una nueva conceptualización sino que además desarrolla un sistema de evaluación para planificar los apoyos y servicios de acuerdo con las necesidades detectadas. Este nuevo enfoque de la AAMR se ha profundizado en años posteriores, dando lugar a un nuevo paradigma sobre discapacidad intelectual, que se concreta en la reformulación que establecen en el 2002 Luckasson y colaboradores.

Todos estos cambios progresivos han supuesto una transformación importante en la política de servicios que tiene que dar respuesta (apoyos, recursos, ayudas) a cada individuo de su comunidad dentro del respeto a las diferencias individuales, del derecho a la igualdad de oportunidades, así como al reconocimiento de capacidades.

La década de los noventa supuso un cambio en la conceptualización de la discapacidad intelectual, y se enfatiza la atención hacia la calidad de vida. Se dice que la calidad de vida para las personas con discapacidad se compone de los mismos factores que para el resto de las personas. La calidad de vida aumenta cuando se da a las personas el poder de participar en decisiones que afectan a sus vidas, y cuando se da una aceptación e integración plena en la comunidad. En definitiva, se experimenta calidad de vida cuando se tienen las mismas oportunidades que el resto de perseguir y lograr metas significativas y se dispone de los apoyos necesarios para que las oportunidades sean reales.

Un indicador fundamental que define el constructo calidad de vida es que el individuo alcance la mayor autonomía personal. Cuando hacemos la reunión a primeros de curso con los padres de los jóvenes usuarios de nuestro proyecto, lo primero que les preguntamos es qué quieren para el futuro de sus hijas e hijos, y la mayoría nos contestan «que lo que buscan es que se valgan por sí mismos», el «valerse por sí mismos» no es otra cosa sino la autonomía personal.

Para que una persona con discapacidad logre esa autonomía tan ansiada por sus progenitores, es necesario que desde el principio de su proceso educativo, los educadores tengamos muy claro el objetivo que queremos lograr, cada actividad propuesta debe tener como fin último la autonomía personal 
adquirida de manera responsable y proporcionando la mayor calidad de vida posible.

Para mejorar la calidad de vida y la autonomía personal de las personas con discapacidad es necesario trabajar no sólo con las personas con discapacidad, sino también en el contexto, lo que supone redefinir y revisar actitudes y percepciones de familiares, profesionales y sociedad en general. En relación a la intervención en los contextos hay que ofrecer apoyos en los mismos, proporcionando oportunidades a las personas con discapacidad y diseñando ámbitos de participación.

La planificación de los servicios y la respuesta que la comunidad debe proporcionar a las personas con discapacidad intelectual tiene como eje el individuo, a partir del cual se organizan las acciones. El objetivo no es crear programas y planes pensados específicamente para personas con discapacidad intelectual, sino que de lo que se trata es de planificar servicios para el conjunto de los ciudadanos, que cuenten con los soportes necesarios para desde el marco de la comunidad responder a las necesidades más específicas. Esta nueva perspectiva en la ordenación y planificación de los servicios está más cerca de posibilitar unas condiciones de vida normales, también para personas con discapacidad intelectual, que es la meta fundamental de nuestro proyecto «Hacia una vida independiente».

\section{Descripción del proyecto de investigación}

Deseamos destacar el carácter innovador del proyecto «Hacia una Vida Independiente». En nuestro país hay muy pocas experiencias en esta línea, las más conocidas y sobre las que nos hemos fundamentado han sido: Proyecto Aura Habitat de Barcelona coordinado por Gloria Canals y el Proyecto Vida Independiente en Murcia coordinado por Nuria Illán².

2. Nuria Illán y Gloria Canals, ambas profesoras de Universidad, iniciaron estos proyectos de vida independiente en España y son las bases en las que asentamos nuestro proyecto. El proyecto Aura Habitat se inició en 2002 y consiste en ofrecer la oportunidad a jóvenes con Síndrome de Down y otros trastornos globales del aprendizaje, de hacer una estancia en un entorno normalizado con jóvenes estudiantes universitarios, aprendiendo a convivir y a hacerse cargo de las responsabilidades que comporta la convivencia. Se trata, pues, de un periodo de residencia en un piso compartido por tres participantes del proyecto Aura y tres estudiantes de psicología, ciencias de la educación o trabajo social, para adquirir unas habilidades y responsabilidades que generalmente son difíciles de adquirir en el hogar, con la familia. La experiencia de vivir en Habitat es el primer paso hacia la autonomía y permite a las familias pensar en el futuro con más tranquilidad comprobando las habilidades y necesidades reales de sus hijas e hijos. 


\subsection{Necesidad de este proyecto en nuestra ciudad, Huesca}

Pensamos que hacerse mayor en casa de los padres es un reto difícil para todos, también para las personas con discapacidad, y optamos por preguntar en primer lugar a los posibles usuarios del proyecto, las/los jóvenes con discapacidad. Nos dimos cuentan de que todas sus respuestas se podrían resumir en dos razonamientos, unos decían que tenían muchos deseos de tener su propia casa como la tienen muchos chicos de su edad. Otros se encontraban muy bien viviendo con sus padres pero tenían ganas de demostrar al mundo que eran capaces de vivir solos y de cuidarse de sí mismos. En las contestaciones de los jóvenes no vimos diferencias por la variable sexo, tanto chicos como chicas querían vivir solos y demostrar al mundo que eran capaces de hacerlo. También preguntamos a sus familias, que en muchos casos mostraban una gran preocupación por la gran dependencia que veían en sus hijos y el miedo de pensar en el día en que ellos fallezcan.

Estudiamos las alternativas de vivienda que existían hasta el momento en nuestra ciudad para las personas con discapacidad, como son los pisos tutelados y las residencias, si bien cumplían una misión muy importante para un sector de esta población, sin embargo creemos que tienen un carácter más paliativo que formativo. La población con discapacidad es muy heterogénea y el Proyecto Vida Independiente posee unas cualidades y características que nos parecen innovadoras y que da respuestas interesantes a esa heterogeneidad.

El proyecto lo entendemos como una oportunidad para que jóvenes con discapacidad puedan aprender a vivir, si lo desean, de manera independiente. Partimos siempre de su voluntad, ningún joven ha entrado al proyecto forzado, partimos de sus intereses y de las ganas de querer aprender a ser autónomos que tengan los jóvenes. El proyecto es un piso de estudiantes, donde viven cuatro jóvenes, dos estudiantes universitarios y dos jóvenes con discapacidad.

\subsection{Personas claves en el proyecto}

\subsubsection{Los estudiantes o responsables}

El papel del alumno de la Facultad que comparte piso con jóvenes con discapacidad es fundamentalmente el de mediador en la actuación del compañero. Su papel asesorado y orientado por el coordinador, va a ser conocer qué es capaz de hacer ese chico/a por su cuenta y qué puede llegar a hacer con su ayuda, de tal forma que tendrá un papel de guía y tutor en el día a día de su compañero, respondiendo fundamentalmente a las demandas de la persona con discapacidad no anticipándose a ellas. 


\subsubsection{Los coordinadores del proyecto}

La coordinación de este proyecto se lleva a cabo por un equipo de profesores pertenecientes a los Departamentos de Psicología y Sociología y de Ciencias de la Educación de la Facultad de Ciencias Humanas y de la Educación de Huesca. Se mantiene una relación de colaboración y una conexión muy estrecha con la Coordinadora de Asociaciones de Personas con Discapacidad de Huesca (CADIS- Huesca).

\subsection{Contenidos del programa}

Los principales contenidos que se trabajan son:

- adquisición y generalización de habilidades de relación interpersonal.

- adquisición y generalización de habilidades de la vida diaria relacionadas con el vestido, el aseo personal y la alimentación.

- desarrollo de la responsabilidad y autonomía en el desempeño de las tareas domésticas.

- desarrollo de la capacidad de emplear el tiempo libre en compañía de personas de su misma edad.

Todas estas adquisiciones, que se han iniciado ya en el marco familiar, tienen en el nuevo contexto la característica esencial de que responderán a la iniciativa personal de las personas con discapacidad.

\subsection{Situación actual}

El proyecto «Hacia una vida independiente» lleva ya tres cursos académicos de andadura, y los resultados obtenidos los consideramos lo suficientemente relevantes como para exponerlos en este artículo por ser una investigación pionera y por representar un paso más en el desarrollo de la inclusión social de las personas con discapacidad intelectual.

Por el proyecto han pasado ya seis usuarios y cuatro estudiantes, nuestra máxima es dar la oportunidad al mayor número de personas, aunque en este artículo sólo nos detendremos en los usuarios del curso 2007-2008.

\subsubsection{La evaluación inicial de las/los jóvenes}

Para valorar la mejora que a lo largo de un curso tienen los usuarios en el piso, pasamos el Inventario de Destrezas Adaptativas (CALS) al principio y al final de la experiencia, que es un instrumento de la Universidad de Deusto de Morreau, Burinniks y Montero, criterial que se aplica de forma individualizada y que consta de 800 destrezas adaptativas relativas a cuatro áreas de 
conocimiento: destrezas de vida personal, destrezas adaptativas de vida en el hogar, destrezas de vida en la comunidad y destrezas laborales (estas últimas no las analizamos). El CALS nos sirve para evaluar la situación inicial de cada joven y para diseñar objetivos de aprendizaje individualizados y hacer el seguimiento de la adquisición de los mismos. No es necesaria formación previa para poderlo cumplimentar, pero sí que es imprescindible que la persona que lo complete haya convivido con los jóvenes varios meses.

El CALS se completa con el ALCS, que es un currículum comprensivo diseñado para facilitar la enseñanza de una destreza específica. Es decir el CALS nos sirve para conseguir la evaluación del joven en un momento determinado y lo aplican los estudiantes universitarios con nuestra ayuda, y a partir de sus necesidades preparamos con ayuda del ALCS objetivos específicos de enseñanza.

Hemos pasado también otras escalas sobre calidad de vida y autodeterminación.

\section{Objetivos}

Los objetivos de nuestra investigación son comprobar si nuestro proyecto favorece:

- la vida independiente,

- la plena autonomía según la posibilidades de cada individuo,

- la autoestima de las personas con discapacidad. Superación personal,

- la calidad de vida de estas personas,

- si hay diferencias de actitud ante las tareas del hogar por razón de sexo

\section{Hipótesis}

A continuación exponemos la hipótesis que nos hemos planteado con este proyecto de investigación.

Hipótesis:

La implementación del programa «Hacia una vida independiente» favorecerá la generalización de habilidades sociales y de autonomía de las personas con discapacidades psíquicas diversas, además de mejorar enormemente su autoestima y su calidad de vida y acercarlas cada vez más a la normalización plena en todos los ámbitos personales y en diferentes contextos sociales independientemente del sexo de los usuarios». 


\section{Diseño metodológico}

Las investigaciones científicas pueden realizarse a partir de metodologías cuantitativas o cualitativas. La primera consiste en el contraste de teorías ya existentes a partir de una serie de hipótesis surgidas de la misma, haciéndose necesario el tener una muestra representativa de la población objeto de estudio. La metodología cualitativa no precisa una muestra representativa, sino una muestra teórica conformada por uno o más casos. Dentro de las metodologías cualitativas el estudio de casos es una estrategia metodológica propia de la investigación científica.

Sabemos que el método de estudio de casos ha sido muy cuestionado por algunos autores, quienes consideran que su prestigio es bajo y no lo perciben como una buena estrategia para realizar investigación científica. Según ellos, el método de estudio de casos presenta problemas de fiabilidad y validez, por lo que son partidarios de utilizar en la investigación científica únicamente métodos cuantitativos.

Sin embargo, el método de estudio de casos es una herramienta valiosa de investigación, y su gran fortaleza se encuentra en que mediante él se puede medir y registrar la conducta de las personas involucradas en el fenómeno estudiado. A esto se suma que en el método de estudio de casos los datos pueden ser obtenidos desde una variedad de fuentes, tanto cualitativas como cuantitativas, por ejemplo a través de registros, entrevistas, observación, documentos, etc.

Yin $^{3}$ argumenta que el método de estudio de casos ha sido una forma esencial de investigación en las ciencias sociales y muy especialmente en educación y considera el método de estudio de casos apropiado para temas que se consideran prácticamente nuevos. Así Eisnhardt ${ }^{4}$ entiende que el estudio de casos es una estrategia de investigación dirigida a comprender las dinámicas presentes en contextos singulares. Y se puede utilizar un único caso o varios, utilizando diferentes métodos de recogida de información con el fin de describir, verificar o generar teoría.

Yin recomienda la utilización de múltiples fuentes de datos y el cumplimiento del principio de triangulación para garantizar la validez interna de la investigación. Esto permitirá verificar si los datos obtenidos a través de las diferentes fuentes de información guardan relación entre sí (principio de triangulación); es decir, si desde diferentes perspectivas convergen los efectos

3. YIn, R. K. Case study research. Desing and methods. London, Ed. Sage Publications, 1994.

4. EISNHARDT K. M. «Building Theories from case study Research». Academy of Management Review, 14, 4 (1989), pp. 532-550. 
explorados en el fenómeno objeto de estudio. En consecuencia, nuestra investigación utiliza diferentes fuentes de información y distintos instrumentos de recolección de información, tales como entrevistas personales no estructuradas, entrevistas personales estructuradas, encuestas por cuestionarios, observación directa no estructurada, revisión de documentos, etc.

Nuestra investigación es pues de carácter cualitativo, y el método utilizado es el estudio de casos, el cual se define como «una descripción intensiva, holística y un análisis de una entidad singular, un fenómeno o unidad social. Los estudios de casos son particulares, descriptivos y heurísticos y se basan en el razonamiento inductivo al manejar múltiples fuentes de datos» ${ }^{5}$.

El estudio de casos como estrategia resultó ser el más coherente con la línea de estudio, y se realizó un estudio para cada una de las personas que convivían en el piso del Proyecto Vida Independiente, con el fin de obtener información desde su propio contexto y significado.

\subsection{Tipo de estudio}

En el momento de emprender una investigación social, es necesario definir los tipos de estudio, que, siguiendo a Dankhe, se pueden dividir en: exploratorios, descriptivos, correlacionales y explicativos. El tipo de estudio dependerá de lo que se quiera investigar y de los objetivos que se pretendan alcanzar en la investigación.

Nuestra investigación es de carácter descriptivo, los estudios descriptivos se entienden como aquellos estudios que buscan especificar las propiedades importantes de personas, grupos, comunidades o cualquier otro fenómeno que sea sometido a análisis. Describir situaciones o eventos, cómo son y cómo se manifiestan determinados fenómenos, se centra en medir con la mayor precisión posible. Requiere considerable conocimiento del área que se investiga para formular las preguntas específicas que busca responder.

Estos estudios nos permiten conocer la perspectiva de los protagonistas de nuestra investigación, de cómo se sienten conviviendo en un piso de estudiantes, de si son más independientes, más autónomos. También nos permite analizar la valoración que hacen sus compañeros de piso, o valorar si los resultados son diferentes dependiendo del sexo.

5. Pérez Serrano, G. Investigación cualitativa: retos e interrogantes I. Métodos. Madrid, La Muralla, 1998, p. 85. 


\subsection{Unidad de estudio}

La unidad de estudio son los usuarios del Proyecto Vida Independiente, fundamentalmente las personas con discapacidad intelectual.

\subsection{Universo}

Son dos jóvenes (un chico y una chica) entre 18 y 30 años, ambos con discapacidad, ella presenta una discapacidad visual asociada a discapacidad intelectual moderada, y él presenta una hemiplejia asociada a discapacidad intelectual.

Los estudiantes han terminado su carrera de maestros y actualmente están estudiando la licenciatura en Educación Física. Son personas que han presentado siempre una especial sensibilidad hacia las personas con discapacidad.

\subsection{Muestra}

La elección de los casos que presentamos en este artículo, así como los casos que pertenecen a nuestra investigación, tienen un carácter teórico puesto que se basan en una elección analítica y no estadística. No se trata de generalizar los resultados en términos estadísticos. La elección de la muestra responde a su capacidad explicativa. El objetivo de nuestra investigación no es generalizar los resultados obtenidos a una población determinada, pero sí dar a conocer nuestra investigación desde un punto de vista teórico-experimental.

Además la muestra utilizada en nuestra investigación es de tipo intencionada, que se entiende como «aquella muestra dónde los sujetos de ésta no son elegidos siguiendo las leyes del azar, sino de alguna forma intencional» ${ }^{6}$. De esta manera, nosotros como investigadores seleccionamos a todos los usuarios del proyecto Vida Independiente, especialmente a los jóvenes que presentaban discapacidad.

\subsection{Técnicas de recogida de datos}

En la metodología de investigación de estudios de casos se utilizan múltiples fuentes de información para obtener los datos que posteriormente son analizados, entre ellas las utilizadas en nuestra investigación han sido:

Revisión de documentos:

En nuestra investigación hemos analizado los diarios que los estudiantes han ido elaborando. También documentación audiovisual, del tipo de

6. RUIZ IGNACIO, O. Metodología de la investigación cualitativa. $2^{a}$ Edición, Bilbao, Universidad de Deusto, 1999. 
fotografías o vídeos grabados de diferentes acontecimientos que se produjeron en el día a día.

Entrevistas o cuestionarios.

En nuestra investigación hemos pasado a los usuarios que presentan algún tipo de discapacidad tres cuestionarios: uno sobre habilidades para la vida diaria (cuestionario que se paso al inicio de la estancia en el piso y justo antes de terminar su estancia) y que luego analizaremos. Es un cuestionario de la Universidad de Deusto denominado «Inventario de destrezas adaptativas CALS».

También se pasaron dos cuestionarios más, uno sobre Calidad de Vida de R. L. Schalock y K, D. keith de 1993 y que han sido adaptadas al castellano por Verdugo, Caballo, Jordán de Urries y Crespo en 1999, y también otro cuestionario sobre autodeterminación con versión para adultos del Instituto Universitario de Integración en la Comunidad INICO de la Universidad de Salamanca.

\section{La observación directa}

La observación por parte de los estudiantes del tipo participante también nos ha arrojado datos de gran interés como posteriormente veremos en los resultados.

El estudio de casos es un método de investigación que recoge datos de diferentes fuentes; como terminamos de describir, debe tener en cuenta tres principios básicos que ayudan a incrementar sustancialmente la calidad y a construir la validez y fiabilidad del caso ${ }^{7}$.

- La triangulación: supone el recoger múltiples medidas sobre el mismo fenómeno. Se utiliza la triangulación como principio que aporta racionalidad y ayuda a conseguir la validez interna y externa de la investigación. La validez interna se consigue cuando para la recogida inicial de datos y para su interpretación se aplican diferentes técnicas como hace un momento hemos descrito, ya que hemos utilizado técnicas como la observación participante, los cuestionarios, los diarios, etc. Y la validez externa se materializa en nuestra investigación en que los datos obtenidos vienen corroborados por la contrastación de recogida de datos de varios investigadores.

- La fiabilidad de la investigación se sustenta en la organización y documentación de datos. Contamos con una base de datos que sustenta nuestro estudio de casos, de tal manera que con los datos que tenemos recogidos, un nuevo investigador podría repetir el estudio llegando a

7. YIN. Op. Cit. 
conclusiones similares. Como dice $\mathrm{Yin}^{8}$, debemos registrar toda la información obtenida con el fin de que si alguien nos audita llegue a los mismos resultados.

- La última recomendación que da Yin ${ }^{9}$ para incrementar la calidad y validez y fiabilidad del método de investigación de casos es el crear una cadena con las evidencias, de tal manera que un observador externo a la investigación que siga la lectura de este artículo pueda ver todos los pasos de la investigación claramente.

\section{Resultados}

A continuación vamos a exponer los resultados obtenidos en la investigación a lo largo del curso escolar 2007-2008. Este año en el proyecto había dos usuarios, una chica a la que llamaremos María y un chico al que llamaremos César. Para ello vamos a analizar cada caso por separado, aunque sin duda en algunas ocasiones no podamos evitar comparar el comportamiento de los jóvenes.

En primer lugar vamos a analizar los resultados que ha obtenido María, a través del Inventario de Destrezas Adaptativas (CALS), este inventario como ya se ha dicho se paso al principio y al final de la estancia en el piso para ver la evolución de los jóvenes y la mejoría que han tenido en las diferentes áreas de intervención. Posteriormente veremos los resultados obtenidos por César.

Para completar la información obtenida a través del inventario, también se ha evaluado la calidad de vida de los usuarios del piso. La escala utilizada para evaluar la calidad de vida puede administrarse a personas con retraso mental que tengan adecuadas habilidades de lenguaje receptivo y expresivo como es el caso de nuestros usuarios. Los evaluadores han sido sensibles a la posibilidad de que estas personas no comprendieran algún ítem o el significado de alguna de las palabras, cuando esto sucedía ellos parafraseaban el ítem para mejorar la comprensión.

Se les pregunta en esta escala sobre la competencia / productividad, en este apartado se hacen diez preguntas relacionadas con el empleo, hay otras diez preguntas sobre autodeterminación / independencia, otras diez sobre satisfacción en la vida, otras diez sobre integración en la comunidad.

Otra evaluación complementaria que también se ha realizado es sobre autodeterminación. Elegimos una escala de autodeterminación diseñada como autoinforme para ser usada por adultos con discapacidad. La escala consta

8. YIN. Op. cit. 1994.

9. Ibídem..

Feminismo/s 13, junio 2009, pp. 207-230 
de 72 ítems y esta dividida en cuatro secciones. Cada sección examina una dimensión de la autodeterminación: Autonomía, Autorregulación, Capacitación Psicológica y Autoconciencia.

Los datos obtenidos en estas escalas complementan los del Inventario de Destrezas Adaptativas y responden a uno de los principios básicos que ayudan a incrementar sustancialmente la calidad y a construir la validez y fiabilidad de método de investigación denominado estudio de casos, que es la triangulación. La triangulación supone el recoger múltiples medidas sobre un mismo fenómeno. Esto nos ayuda a conseguir una mayor validez interna. A la vez la validez externa se materializa en nuestra investigación en que los datos obtenidos vienen corroborados por la contrastación de recogida de datos de los dos estudiantes y por el análisis de los mismos de todos los investigadores que formamos parte de este proyecto.

A continuación se ven los resultados de los dos sujetos de los cuales se ha hecho el estudio:

La tabla que vemos, muestra los resultados obtenidos por María. y su evolución en las diferentes áreas. En la primera fila de la tabla se han trascrito las áreas a evaluar. En la primera fila se colocan las valoraciones obtenidas para cada área al inicio de la estancia de la joven en el piso y la segunda fila representa la puntuación que en cada área obtiene la joven después de haber convivido en el proyecto a lo largo de los nueve meses.

Tabla 1 (Ver Anexos)

Tabla 2 (Ver Anexos)

Tabla 3 (Ver Anexos)

María ha mejorado mucho al vivir en el piso. Las áreas en las que ha tenido una mejora más notable han sido el área de ocio en el hogar, ocio comunitario, participación en la comunidad y búsqueda de empleo. En el resto de áreas relacionadas con las tareas del hogar, su evolución también ha sido muy positiva. María ya había realizado en su casa algunas de estas tareas por lo que su evaluación inicial en algunas de las áreas da resultados iniciales altos que sin duda, como se puede ver en la gráfica, han evolucionado de manera todavía más positiva a lo largo de su estancia en el proyecto. Además María tiene un gran interés y motivación por todas las tareas del hogar, disfruta poniendo una lavadora, planchando, haciendo la comida, barriendo, etc.

En el área de ocio, María ha aprendido a disfrutar de actividades con sus compañeros, ha encontrado el gusto en actividades compartidas y ha pasado de realizar la mayoría de las actividades de manera individual a compartirlas con sus amigos tanto dentro como fuera de la casa. En la comunidad ha mejorado su participación, ha aprendido a solicitar de manera adecuada comida 
en un establecimiento público, sabe usar el teléfono para ponerse en contacto con alguien, abre únicamente su propia correspondencia, ha aprendido a seleccionar una comida en la carta de un restaurante, a esperar su turno en un establecimiento público, etc. En el tema de búsqueda de empleo, igual que César, ha aprendido a llegar puntual y adecuadamente vestida a una entrevista, a cumplimentar una solicitud de empleo, a presentar correctamente su currículum y a pedir carta de recomendaciones a una persona que conoce.

En el resto de las áreas también ha mejorado mucho; en el cuidado de su salud, María ha aprendido a hacer ejercicio con regularidad para mantenerse en forma, sabe que debe leer las instrucciones del prospecto de un medicamento antes de tomárselo, qué alimentos le convienen y cuáles no, etc. En el cuidado de la ropa, actualmente es capaz de limpiarse los zapatos, de guardar la ropa de fuera de temporada en otro lugar, de separar la ropa que se lava a máquina de la que tiene lavado en seco o a mano y también ha aprendido a planchar cualquier tipo de prenda. En el área de socialización, ha mejorado en el ser capaz de esperar a que los demás hayan acabado de hablar antes de empezar a hacerlo ella, es decir, actualmente no interrumpe a los demás cuando están hablando. En el tema del vestido y la presencia física, ha mejorado mucho. Actualmente, antes de salir de casa comprueba su apariencia ante el espejo, y realiza los ajustes y retoques oportunos en su ropa. En la planificación y preparación de las comidas también se ha visto una mejoría muy importante. Ha aprendido a lavarse y secarse las manos antes de manipular cualquier alimento, a abrir latas con un aparato manual o eléctrico, sabe hacer café para todos, es capaz de seleccionar la temperatura de la placa que desea, saca los alimentos del congelador a tiempo para descongelarlos, mide la cantidad de ingredientes que necesita para la elaboración de una receta, pone a remojo con antelación las legumbres que va a cocinar, es capaz de programar un temporizador para que suene la alarma cuando la comida está lista, utiliza adecuadamente la olla a presión, sabe utilizar el horno, es capaz de preparar una comida con los restos que quedaron de las anteriores, sabe preparar una comida completa para cuatro personas y servirla, es capaz de planificar comidas equilibradas para toda una semana incluyendo alimentos de las cuatro categorías (lácteos, fruta-verdura, carne-pescado y cereales).

En el tema de seguridad en el hogar, ha adquirido una serie de comportamientos de precaución que le permiten ser autónoma, de tal manera que respeta e interpreta correctamente signos gráficos de peligrosidad, sabe llamar algún gremio cuando algo se estropea en la casa, igual que César tiene precaución para introducir los alimentos en el aceite caliente, retira los productos 
inflamables de la cercanía del fuego y coloca el mango de las cacerolas hacia el interior de la cocina cuando está guisando.

En la escala que se le ha pasado para evaluar su calidad de vida, se observa que María está deseando encontrar un trabajo, actualmente no tiene ninguno y su tiempo lo dedica a hacer cursos de formación. Le gustaría mucho trabajar la cerámica, sus últimos cursos de formación han ido orientados a esta área y podemos asegurar que tiene unas manos que son capaces de hacer piezas geniales.

María, en el área de autodeterminación o independencia, ha reflejado estar satisfecha, dice, entre otras cosas, que es ella quien decide cómo gastar su dinero, que tiene llave de su propia casa y que le gustaría mucho seguir viviendo con los amigos del piso o en un piso sola.

En el área de integración en la comunidad, como César, reconoce participar en tres clubes o asociaciones, y está satisfecha con ello. Suelen invitar a amigos a su casa, participa de manera activa en actividades de ocio. Reconoce que su vida es como la de todos las demás, aunque no cree que nunca contraiga matrimonio ni tenga pareja.

En el piso ha aprendido también a expresar sus ideas, aunque estas sean diferentes a las de los demás, a tomar sus propias decisiones, a hacer amigos, a hacer buenas elecciones, y a decirle a alguien que algo no le ha gustado o incluso que le ha molestado.

Tabla 4 (Ver Anexos)

Tabla 5 (Ver Anexos)

Tabla 6 (Ver Anexos)

Aunque los mejores resultados en el Inventario de Destrezas Adaptativas sean los obtenidos por María, sin embargo la evolución más positiva y más ascendente la ha tenido César, él era al entrar en el proyecto mucho más dependiente y menos autónomo que María en todo lo relacionado con las tareas de realización de comidas, higiene, socialización, vestido, cuidado de la salud, cuidado de la ropa, limpieza del hogar, planificación de comidas y su preparación, en ocio en el hogar, en mantenimiento del hogar, etc. Tenían puntuaciones iniciales muy parecidas en interacción social, en seguridad en el hogar, en seguridad comunitaria, ocio comunitario, participación en la comunidad y búsqueda de empleo.

Cada uno ha adquirido niveles de desenvolvimiento muy interesantes en prácticamente todas las áreas. En César las áreas que inicialmente estaban más flojas y que por lo tanto hemos tenido que trabajar más han sido las áreas de higiene personal, cuidado de la ropa, limpieza del hogar, ocio en el hogar, 
mantenimiento del hogar, ocio comunitario, manejo del dinero y búsqueda de empleo.

Las áreas que más han mejorado en César después de pasar por la experiencia del piso han sido: la comida, la higiene personal, la limpieza del hogar, la planificación y preparación de una comida, ocio en el hogar, interacción social, seguridad en el hogar, ocio comunitario y búsqueda de empleo.

El área que sin duda más ha mejorado es el área de la higiene personal, César sabía lo que debía hacer para estar aseado, pero no tenía rutinas adquiridas que realizara de manera frecuente. Con la estancia de César en el piso, hemos conseguido que cambiara de muda diariamente, que se duchara prácticamente todos los días y que se cepillara los dientes después de cada comida y antes de irse a la cama. Además ha aprendido a lavarse el pelo y secárselo de manera autónoma. Desde nuestro modesto punto de vista, la mejora en el área de la higiene lo ha ayudado a integrarse mejor con los compañeros tanto del piso como fuera de él.

También ha progresado mucho en el área de limpieza y organización del hogar. César, en casa de sus padres no realizaba ninguna de las tareas del hogar, suponemos que por ser chico, y tampoco eran tareas que le gustaran demasiado, pero en el piso ha realizado todas las tareas de una manera muy autónoma, ha barrido, ha fregado, ha puesto el lavaplatos, la lavadora, ha tendido, ha planchado, ha limpiado cristales, se ha hecho la cama, ha limpiado su habitación, ha limpiado el polvo, ha sabido descongelar y limpiar el frigorífico, también ha sido capaz de limpiar el horno, ha cocinado, etc. Ha sido ésta una de las áreas en la que más ha evolucionado y la actitud que ha tenido hacia estas tareas ha sido muy positiva.

César ha mejorado también mucho en el área de interacción social, el hecho de vivir cuatro personas de edades similares en un piso, ayuda mucho a aprender a convivir, a respetar las diferencias, a compartir todo lo que hay en el piso, a adquirir habilidades sociales para que la convivencia sea lo más llevadera posible. César, al finalizar su estancia en el piso, es capaz de ofrecer y dar su apoyo cuando alguien lo necesita, de defenderse cuando alguien intenta agredirlo, de preguntar a los compañeros si puede participar en alguna actividad común, etc.

También ha mejorado mucho en precaución y seguridad en el hogar como podemos ver en los resultados obtenidos y en las gráficas. Cesar tiene actualmente mucho cuidado cuando introduce alimentos en la sartén con aceite, siempre coloca el mango de la sartén o cazuelas hacia el interior de la cocina cuando está guisando, también ha aprendido a no tener productos inflamables cerca de los fogones. La evolución en ésta y otras áreas nos permite decir 
que tanto Cesar como María, están preparados para vivir solos después de haber estado un curso escolar en el piso de entrenamiento, tan solo necesitarían el apoyo de un nuevo perfil profesional que sería el «mediador del hogar». Al principio el apoyo del mediador tendría que ser mayor, pero poco a poco se reduciría a unas visitas para ver cómo va el funcionamiento y el día a día en el nuevo hogar.

El ocio en el hogar y el ocio comunitario también han sido dos áreas que han mejorado muchísimo en Cesar. Desde que está viviendo en el piso, César ha hecho actividades con el resto de los usuarios del piso, como jugar a cartas, cocinar, etc. además en varias ocasiones ha sido capaz de invitar a amigos a la casa y de ocuparse de ellos, ofrecerles algo de beber y comer. También César ha hecho actividades físicas con regularidad en casa, tablas de gimnasia, etc. Al final de la estancia, César ha empleado su tiempo libre en las actividades que le interesan, es capaz de planificar los fines de semana con actividades que le gustan hacer, participa en actividades organizadas por su comunidad, llama por teléfono a sus amigos. En pocas palabras que su ocio en el hogar y en la comunidad ha cambiado mucho desde su paso por el proyecto Vida Independiente, ha pasado de ser únicamente el ver la televisión o escuchar música él solo, a ser capaz de compartir ocio con otros compañeros en el hogar y realizar actividades muy diferentes.

Ninguno de los dos chicos estaba trabajando en empresa ordinaria, pero en el período de tiempo que han vivido en el piso les han llegado algunas ofertas de trabajo, aunque no se ha trabajado directamente esta área, nos hemos esforzado mucho en reforzar lo importante que es la presencia física en una entrevista de trabajo, la preparación del currículum etc. Al terminar la estancia en el piso, los jóvenes han mejorado mucho en esta área, llegaban puntuales y adecuadamente vestidos a las entrevistas de trabajo, sabían cumplimentar una solicitud de empleo, pedían cartas de recomendación a una persona que conocían, sabían preparar el currículum personal y presentarlo.

Al finalizar su estancia en el piso se les ha aplicado una escala para evaluar como se sentían ellos en relación con la calidad de vida. César se sentía pleno, se sentía muy competitivo y productivo, él estaba trabajando en un taller protegido, pero se sentía muy bueno en el taller, muy satisfecho con las habilidades y experiencias que había adquirido en el trabajo, sin embargo él estaba deseando cambiar de trabajo, le gustaría ser funcionario y se ha apuntado a unas oposiciones públicas.

En relación con su autonomía, si bien reconoce que otra persona decidió por él para estar en el taller, es él el que decide en qué gastar su dinero, actualmente dice que es él el que decide acerca de lo que hace cada día, quien tiene 
la llave de su casa, en general se siente autónomo aunque entiende que para algunas cosas necesita supervisión y cierta mediación.

En el área de satisfacción personal, él dice estar muy satisfecho de vivir en el piso de vida independiente, dice no sentirse solo y divertirse y aprender muchas cosas nuevas, además de haber hecho unos nuevos amigos, como si fueran su segunda familia.

En el área de integración en la comunidad, dice que pertenece a más de tres asociaciones o clubes de su comunidad y que está muy satisfecho con los mismos, tiene muy buena relación con sus vecinos y algunas veces vienen amigos a visitarlo y en varias ocasiones participa en actividades como fiestas, bailes, conciertos. En general esta muy contento con su vida, con sus amigos, con su familia, aunque dice que en el tema de pareja o contraer matrimonio, tiene pocas oportunidades de conocer a alguien y cree que no le dejarían contraer matrimonio, además piensa que su situación laboral podría mejorar mucho.

\section{Conclusiones}

Podemos concluir que el programa de transición a la vida independiente que hemos diseñado en Huesca desde la Facultad de Ciencias Humanas y de la Educación, favorece las generalización de habilidades sociales y la autonomía de las personas con discapacidad intelectual, además mejora su autoestima y su calidad de vida y las acerca cada vez más a la normalización plena en todos los ámbitos personales, en diferentes contextos sociales independientemente del sexo de los usuarios.

Podemos confirmar que el sexo no es una variable determinante en este estudio, que tanto chicos como chicas mejoran su habilidad para la vida diaria al pasar por el proyecto, pero sí que hemos observado cómo las condiciones iniciales con las que llegan al proyecto las chicas y los chicos son diferentes. Las madres de las chicas con discapacidad tienen una gran preocupación porque las jóvenes aprendan las tareas del hogar y les enseñan a hacerlas en casa, cuando llegan al proyecto ya conocen muchas de esas tareas que se trabajan en nuestra investigación. Además, al regresar al hogar familiar, las madres se encargan de que pongan en práctica todo lo aprendido en el piso y así generalicen sus aprendizajes. En cambio, en la mayoría de los jóvenes con discapacidad, el interés de las madres no es tanto el que aprendan a desenvolverse en la casa y aprendan las tareas del hogar, sino la de que puedan integrarse laboralmente en una empresa ordinaria a trabajar. La mayoría de los jóvenes que entran al piso no han hecho las tareas del hogar, ni siquiera en su casa se hacían su cama, las madres nos dicen que para hacer las tareas del hogar ya están ellas. Además, al regresar del proyecto de nuevo a su hogar, los chicos 
no ponen en marcha los aprendizajes adquiridos en el piso y por lo tanto no generalizan sus aprendizajes y el beneficio que sacan de pasar por el proyecto vida independiente es mucho menor.

\section{Referencias bibliográficas}

Agudo, A.L. Historia de las deficiencias. Madrid, Escuela Libre Editorial, 1995.

EISNHARDT, K M. «Building Theories from case study Research», Academy of Management Review, 14, 4 (1989), pp. 532-550.

Gilman, C.J., Morreau, L.E., Bruininks, R.H., Anderson, J.L., Montero, D. Y UnAmunZaGa, E. Currículum de destrezas adaptativas (ALSC). Ediciones Mensajero, S.A. Bilbao, 2002.

LuCKASSON, R. et al. Mental Retardation: Definition, Clasification and Systems of Support. Washington, AAMR, 2002. (Traducción al castellano: Retraso mental: Definición, Clasificación y sistemas de apoyo, 1997).

Morreau, L.E., BruininKs, R.H. y MONTERO, D. Inventario de destrezas adaptativas (CALS). Ediciones Mensajero, S.A. Bilbao, 2002.

PéREZ SERRANO, G. Investigación cualitativa: retos e interrogantes I. Métodos. Madrid, La Muralla, 1998.

- Investigación cualitativa: retos e interrogantes II. Métodos. Madrid, La Muralla, 1998.

Ruiz Ignacio, O. Metodología de la investigación cualitativa. $2^{a}$ Edición. Bilbao, Universidad de Deusto, 1999.

SCHALOCK, R.I. Quality of Life. Vol I. Washington, AAMR, 1996.

- «Can the concept of quality of life make a difference?», 2000.

SCHALOCK, R.L. y VERDUGO, M.A. Handbook on Quality of life for Human Service. Practitioners. Washington D.C, American Association on Mental Retardation, 2002.

SCHERENBERGER, R.C. Historia del retraso mental. San Sebastián, SIIS, 1984.

- Mental retardation. A quarter of century of promise. Baltimore, Brookes, 1987.

Verdugo Alonso, M.A. (dir). Cómo mejorar la calidad de vida de las personas con discapacidad. Instrumentos y estrategias de evaluación. Salamanca, AMARU, 2006.

- «Las personas con retraso mental». En Verdugo Alonso (dir). Personas con discapacidad. Perspectivas Psicopedagógicas y rehabilitadotas siglo XXI. De España Editores, 1995, pp. 515-551.

YIN, R. K. Case study research. Desing and methods. London, Sage Publications, 1994. 


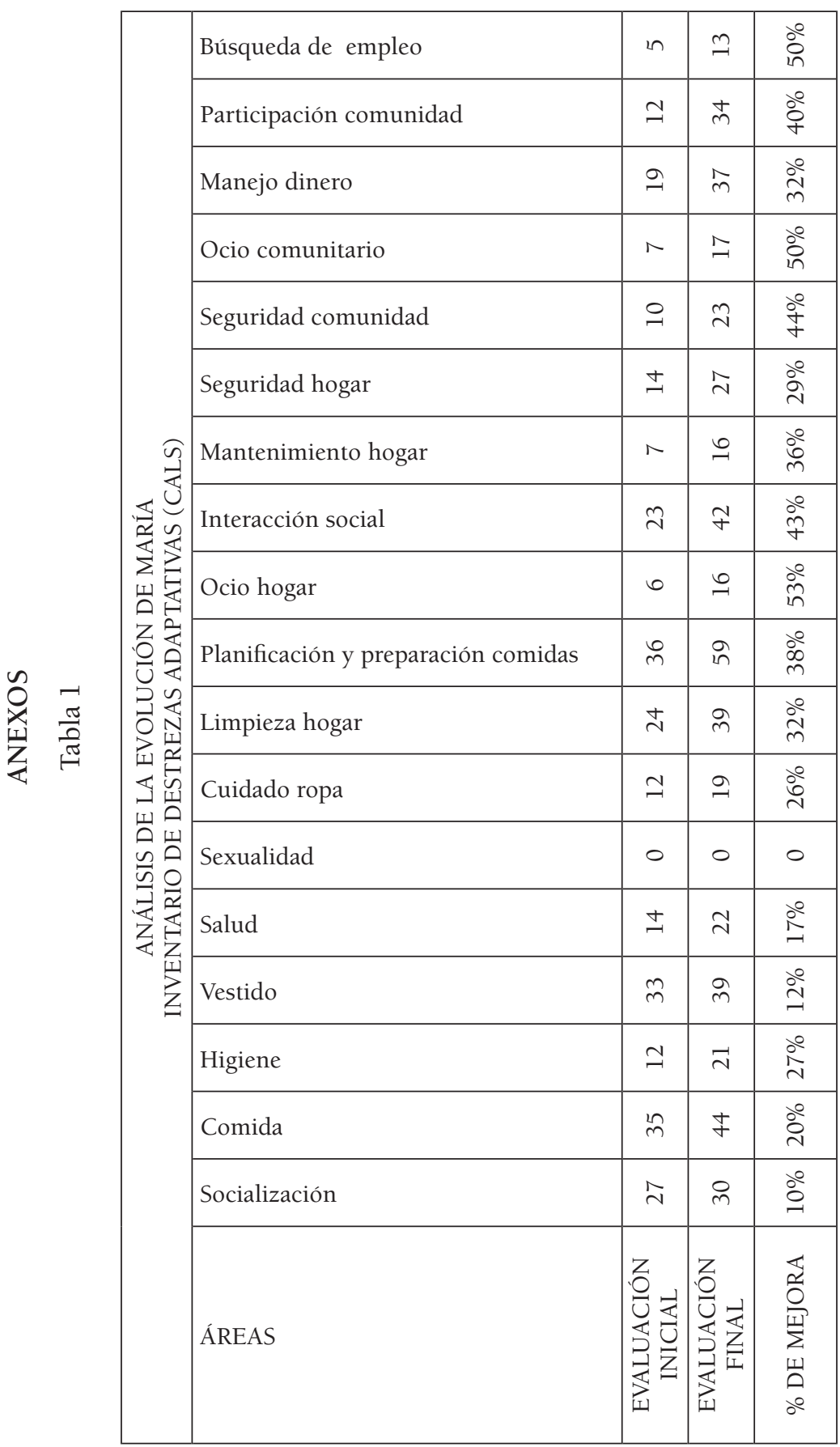

Feminismo/s 13, junio 2009, pp. 207-230 
Tabla 2

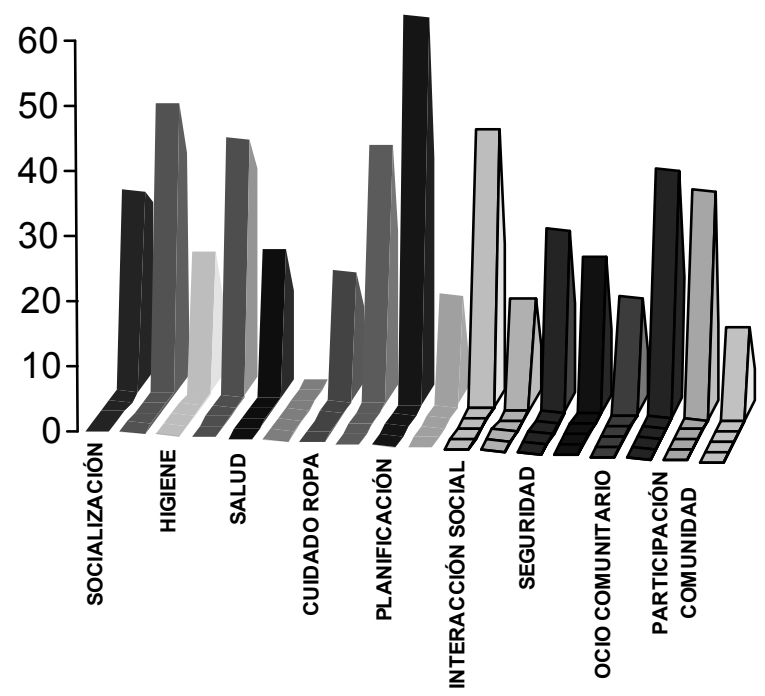

Tabla 3

EVALUACIÓN DE LA CALIDAD DE VIDA DE MARÍA

\begin{tabular}{|l|c|c|}
\hline ESCALA & Calificación & $\begin{array}{c}\text { \% valoración personal } \\
\text { de su calidad de vida }\end{array}$ \\
\hline Competencia / productividad & 14 & $47 \%$ \\
\hline Autodeterminación / independencia & 25 & $83 \%$ \\
\hline Satisfacción & 26 & $87 \%$ \\
\hline $\begin{array}{l}\text { Pertenencia Social / Integración en } \\
\text { la Comunidad }\end{array}$ & 22 & $73 \%$ \\
\hline PUNTUACIÓN TOTAL & 87 & $73 \%$ \\
\hline
\end{tabular}




\begin{tabular}{|c|c|c|c|c|}
\hline \multirow{19}{*}{ 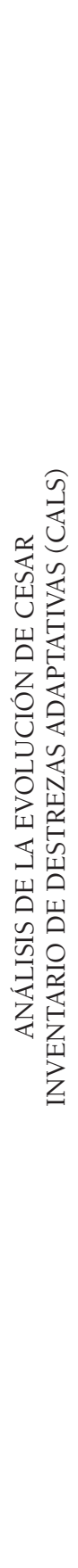 } & Búsqueda de empleo & + & $\stackrel{ }{=}$ & $\begin{array}{l}\stackrel{\circ}{\infty} \\
\infty \\
m\end{array}$ \\
\hline & Participación comunidad & $\underline{n}$ & $\stackrel{\infty}{\sim}$ & $\frac{\stackrel{2}{N}}{\sqrt{2}}$ \\
\hline & Manejo dinero & + & ปี & $\stackrel{\circ}{m}$ \\
\hline & Ocio comunitario & 0 & $\stackrel{0}{-}$ & 号 \\
\hline & Seguridad comunidad & I & $\vec{\sim}$ & $\stackrel{\circ}{\circ}$ \\
\hline & Seguridad hogar & $\exists$ & શे & $\stackrel{\circ}{\stackrel{一}{F}}$ \\
\hline & Mantenimiento hogar & N & + & $\triangleright_{\infty}^{\circ}$ \\
\hline & Interacción social & $\stackrel{\Re}{\sim}$ & ㅇ & ஓे \\
\hline & Ocio hogar & N & $\exists$ & $\stackrel{\circ}{\circ}$ \\
\hline & Planificación y preparación comidas & $\stackrel{\theta}{\theta}$ & $\stackrel{n}{f}$ & $\stackrel{\circ}{\stackrel{+}{+}}$ \\
\hline & Limpieza hogar & $n$ & Әे & ৯̊ \\
\hline & Cuidado ropa & 6 & $\underline{n}$ & 융 \\
\hline & Sexualidad & $\circ$ & $\circ$ & $\circ$ \\
\hline & Salud & $\exists$ & 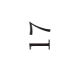 & 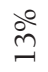 \\
\hline & Vestido & $\vec{\sim}$ & 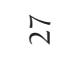 & 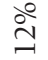 \\
\hline & Higiene & - & \pm & ஓे \\
\hline & Comida & $\vec{\sim}$ & $\stackrel{\sim}{t}$ & 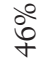 \\
\hline & Socialización & $\stackrel{0}{-1}$ & $\stackrel{\imath}{2}$ & \begin{tabular}{l}
$\stackrel{0}{\circ}$ \\
$\stackrel{\text { \} }{ }}$ \\
\hline & ÁREAS & 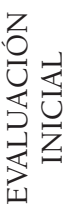 & 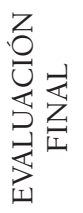 & 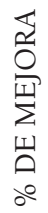 \\
\hline
\end{tabular}
\end{tabular}

Feminismo/s 13, junio 2009, pp. 207-230 
Tabla 5

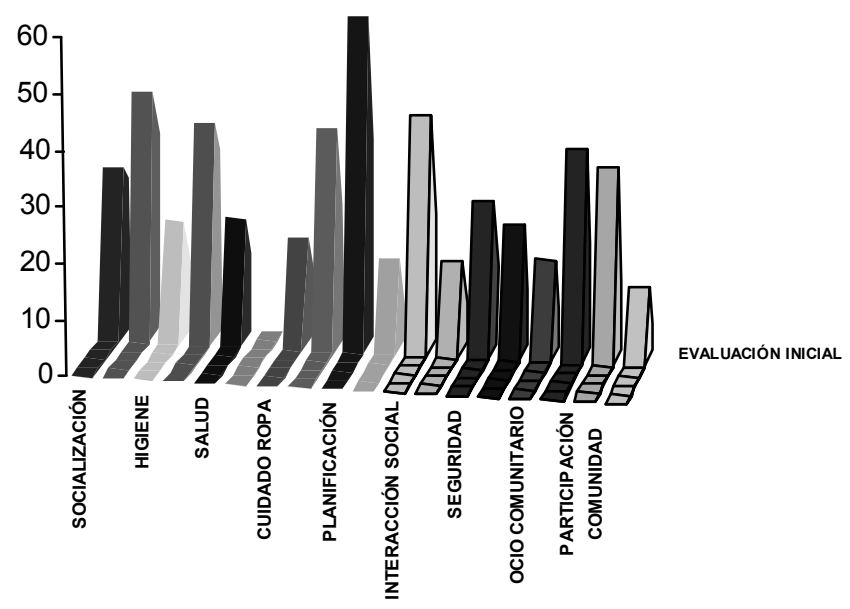

Tabla 6

EVALUACIÓN DE LA CALIDAD DE VIDA DE CÉSAR

\begin{tabular}{|l|c|c|}
\hline ESCALA & Calificación & $\begin{array}{c}\text { \% valoración personal } \\
\text { de su calidad de vida }\end{array}$ \\
\hline Competencia / productividad & 29 & $97 \%$ \\
\hline Autodeterminación / independencia & 26 & $87 \%$ \\
\hline Satisfacción & 26 & $87 \%$ \\
\hline $\begin{array}{l}\text { Pertenencia Social / Integración en la } \\
\text { Comunidad }\end{array}$ & 27 & $90 \%$ \\
\hline PUNTUACIÓN TOTAL & 108 & $90 \%$ \\
\hline
\end{tabular}

